\title{
A REVISTA GALEGA DE ECONOMÍA E OUTRAS REVISTAS DE INVESTIGACIÓN ECONÓMICA PUBLICADAS EN GALICIA, 2001-2018 \\ GUISÁN, María-Carmen
} Catedrática Xubilada de Economía Aplicada (Econometría) da USC Profesora Ad Honorem da USC Directora da RGE en 2014-2018 e-mail: mcarmen.guisan@usc.es

Resumo: Este informe presenta as etapas da Revista Galega de Economía (RGE), logo de cumprir máis de 25 anos de existencia, o que a converte na revista galega de Economía de maior duración. Tamén incluímos información sobre outras revistas de investigación económica publicadas en Galicia, sobre todo as enfocadas cara o desenvolvemento económico, as publicadas no período 2001-2018 e dispoñibles na Internet. A sección 1 fai unha referencia ás tres etapas da RGE: de 1992-1999, de 2000-2013 e de 2014-2018. A segunda etapa supón un avance importante na internacionalización da revista, especialmente a través da inclusión na base RedAlyc dende o ano 2000, e Ideas.Repec dende o ano 2012, sen perder unha elevada adicación á economía galega. A terceira etapa avanza na internacionalización, tamén a través doutros eidos en lingua inglesa, e segue cunha intensa adicación á economía de Galicia e ao desenvolvemento rexional de Europa. A sección 2 achega datos sobre o grao de difusión electrónica dos artigos da RGE, destacando un grande avance no período 2014-2018, tanto en lectores como en citas, e a importante difusión a través de Redalyc. A sección 3 fai referencia a outras revistas de investigación económica publicadas en Galicia e incluídas en selectos índices internacionais. A sección 4 realiza unha breve referencia a publicacións relacionadas coa investigación económica de Galicia no período 1992-2018 e a súa difusión social a través de libros, documentos e Blogs académicos.

Palabras chave: Revistas de Investigación Económica Aplicada, Galicia, España, RedAlyc, Ideas-Repec, Scopus, Divulgación social da investigación económica, Blogs académicos Title: THE REVIEW OF GALICIAN ECONOMY (RGE) AND OTHER JOURNALS OF ECONOMIC RESEARCH PUBLISHED IN GALICIA, 2001-2018

Summary: This report contains selected information on the Review of Galician Economy (RGE), after more than 25 years of existence, which makes it the longest-running Galician Economic Research journal. We also include information on other economic research journals published in Galicia, especially those focused on economic development, published in the period 2001-2018 and available on line. Section 1 makes a reference to three stages of the RGE: 1992-1999, 2000-2013 and 2014-2018. The second stage represents an important advance in the internationalization of the magazine, with its inclusion in RedAlyc since 2000 and in Ideas.Repec since 2012, keeping a high dedication to the Galician economy. The third stage progresses in internationalization, also reaching exposure in English, and continues with an intense dedication to the economy of Galicia and the regional development of Europe. Section 2 provides data on the degree of electronic dissemination of the articles of the RGE, in particular, a considerable increase in the period 2014-2018, both in the number of readers and citations, and the important promotion through Redalyc. Section 3 refers to other economic research journals published in Galicia and included in prestigeous international indexes. Section 4 makes a brief reference to other publications related to economic research in Galicia and its social exposure.

Keywords: Applied Economics Research Journals, Galicia, Spain, RedAlyc, Ideas.Repec, Scopus, Social Dissemination of Economics Research, Academic Blogs.

JEL Codes: A11, A13, 018, 052 


\section{Revista Galega de Economía (RGE): Etapas e Consello de Redacción}

Etapas da Revista Galega de Economía

A Revista Galega de Economía (RGE) foi impulsada no período 1990-1992 polo Profesor Luis Caramés Viéitez, Catedrático e daquela Decano da facultade de Económicas da USC, e contou co apoio da Xunta de Galicia e dos fondos europeos. Logo da aprobación do número 0, a Revista evolucionou conforme ás seguintes etapas, mantendo o seu forte vencello coa economía galega e impulsando a internacionalización dalgúns contidos: a partir da Etapa 2 ampliando a difusión en América Latina e a partir da etapa 3 aumentando as colaboracións en inglés.

Etapa 1. De 1992-1999: Os artigos están dispoñibles en galego, con algunhas achegas noutras linguas, e refírense á economía de Galicia.

Etapa 2. De 2000-2013: Amplíase a publicación a dúas versións, en galego e en castelán. A difusión en castelán, no conxunto de España e en América Latina, incrementa o número de artigos referidos a eses eidos. A revista é aceptada en RedAlyc, o índice SCOPUS de Elsevier, Ideas.Repec e outros prestixiosos índices e bases académicas.

Etapa 3. A partir de 2014: Continúa a publicación bilingüe en galego e castelán, e aumenta o número de artigos en inglés cos obxectivos de aumentar a presenza de perspectivas de desenvolvemento mundial, a difusión europea e mundial de investigacións de economía internacional realizadas en Galicia e de contribuír a que a revista sexa consultada e citada nas bases internacionais.

Sobre os artigos publicados na RGE suxerimos a lectura do traballo de Barreiro (2007) para o período 1992-2006.

\section{Consello de Redacción}

A relación completa dos membros do Consello de Redacción (CR) e do Consello Científico (CC) do período 1992-2006, figura no Suplemento da RGE ao Volumen 16-1 do ano 2007 e os dos anos posteriores figura na edición impresa de cada ano. Os equipos directivos indícanse na seguinte táboa, por orde cronolóxica.

Táboa 1. Membros do equipo Directivo do Consello de Redacción da RGE

\begin{tabular}{|l|l|l|}
\hline Anos & CR: Dirección & Secretario/a do CR \\
\hline $1990-1991$ & Coordinador e promotor: Luis Caramés Viéitez & Edelmira Neira Fontela \\
\hline $1992-1994$ & Coordinador: Manuel Castro Cotón & Pilar Bello Martín Crespo \\
\hline $1995-1999$ & Coordinador: José Alberto Díez de Castro & Pilar Bello Martín Crespo \\
\hline $2000-2005$ & Coordinador: Juan Piñeiro Chousa & $\begin{array}{l}\text { Xosé Antón Rodríguez } \\
\text { González (1999-2005) }\end{array}$ \\
\hline 2005 & Director: Andrés S. Suárez Suárez & Isidro Frías Pinedo \\
\hline $2005-2013$ & $\begin{array}{l}\text { Director: Fausto Dopico Gutiérrez del Arroyo } \\
\text { Co-Director: Jaime Gil Aluja }\end{array}$ & Ana Iglesias Casal \\
\hline $2014-2018$ & $\begin{array}{l}\text { Coordinadora: Emilia Vázquez Rozas } \\
\text { Directora: Ma Carmen Guisán Seijas } \\
\text { Co-Director: Jaime Gil Aluja } \\
\text { Subdirector: Isidro Frías Pinedo }\end{array}$ & $\begin{array}{l}\text { Pilar Expósito Díaz } \\
\text { Ana Iglesias Casal }\end{array}$ \\
\hline
\end{tabular}

Fonte: Elaboración a partir do Suplemento da RGE, Vol. 16-1 do ano 2007 e información posterior da Revista. 


\section{Participación feminina no CR e no CC}

Compróbase un incremento da participación feminina en ámbolos dous consellos. Comparando os datos do período 1992-2006 cos do ano 2018, observamos un incremento do número de mulleres no Consello de Redacción (CR) pasando de 2 mulleres nun conxunto de 23 membros, o que supoñía un $9 \%$ no devandito período inicial, a 8 de 33 membros no ano 2018 (24\%). Polo que respecta ao Consello Científico (CC) tamén se incrementou a participación feminina pasando do 15\% ao 19\%. É desexable continuar o incremento para acadar unha situación máis representativa da participación feminina na investigación económica, a cal no caso de Galicia supera o 40\% como se indica en Guisán (2018).

\section{Difusión da RGE en Galicia e en bases internacionais, período 2014-2018}

É unha Revista en acceso aberto cun elevado número de lectores. A difusión da revista na Internet ten miles de descargas dos seus artigos xa que se difunde a través de bases internacionais. Nas seguintes táboas indicamos a evolución das descargas anuais e mensuais na Web da USC e nas bases RedAlyc e Ideas.Repec. Tamén indicamos estatísticas de citas bibliográficas en SCIMAGO Journal Ranking (SJR) correspondente ás revistas do prestixioso índice SCOPUS de Elsevier.

Táboa 2. Estatísticas de acceso á RGE no SPIC da USC

\begin{tabular}{|c|c|c|}
\hline Ano & Descargas en 365 días & Media mensual \\
\hline 2014 & 2590 & 216 \\
\hline 2015 & 10110 & 842 \\
\hline 2016 & 9509 & 792 \\
\hline 2017 & 10163 & 847 \\
\hline 2018 & 16453 & 1371 \\
\hline
\end{tabular}

Fonte: Elaborado por M.C. Guisán a partir de datos de acceso diario proporcionados polo Servizo de Publicacións e Intercambio Científico (SPIC) da Universidade de Santiago de Compostela (USC). Nota: A cifra de 2018 é provisional, baseada nas 15146 descargas de 336 días, aplicando ao resto de días do ano a media diaria do período con datos dispoñibles.

O contador de accesos na Web do Servizo de Publicacións da USC evolucionou positivamente no período 2014-2018, pasando dunha media mensual de 216 descargas en 2014 a unha media mensual de 1371 descargas no ano 2018. Non sabemos o número de artigos consultados, xa que cada acceso á Web da RGE pode supoñer un ou máis artigos consultados.

No caso da base internacional de América Latina e o Caribe, RedAlyc, a estatística proporciona datos mensuais de descargas de artigos.

Nesta base Redalyc están incluídos 354 artigos da RGE no período 2000-2017, que corresponden a autores de 119 institucións de 24 países. 0 número de descargas anuais de artigos da RGE foi de 1866 no ano 2013 e aumentou ata chegar a 7102 no ano 2017 . As descargas son superiores á media das revistas de Economía e Finanzas da devandita base.

$\mathrm{Na}$ entrada no 18 do Blog de Economía Internacional da EAAEDS, citado ao final da bibliografía, inclúese un informe sobre revistas españolas e latinoamericanas na base RedAdlyc e noutros catálogos. Das 1500 revistas de Economía do Directorio de Latindex, só un número de preto de 300 revistas (20\%) de América Latina, España e Portugal pertencen ao Catálolgo seleccionado de Latindex, e desas 300 só 43 se incluían no ano 2011 en RedAlyc (só o 14\% do Catálogo Seleccionado de Latindex e só o 2,8\% das 1500 revistas do Directorio de Latindex). Entre as revistas españolas do Catálogo Seleccionado 
de Latindex nese ano (arredor de 150) só 13 foron seleccionadas en RedAlyc (menos do 105). Galicia, con 3 revistas, achegaba no ano 2011 o 23\% das revistas españolas na devandita base. As revistas publicadas en Galicia incluídas na devandita base no ano 2011 eran a Revista Galega de Economía (RGE), Estudios Económicos de Desarrollo Internacional (EEDI) e Urban Public Economic Review (UPER).

Táboa 3. Descargas mensuais da Revista Galega de Economía en Redalyc

\begin{tabular}{|l|c|c|c|c|c|}
\hline \multicolumn{1}{|c|}{ Mes } & 2013 & 2014 & 2015 & 2016 & 2017 \\
\hline Xaneiro & 2147 & 1737 & 2800 & 2202 & 7959 \\
\hline Febreiro & 764 & 2281 & 1811 & 3008 & 9993 \\
\hline Marzo & 1479 & 2564 & 2590 & 3144 & 9278 \\
\hline Abril & 2756 & 2327 & 1969 & 5802 & 7305 \\
\hline Maio & 1895 & 2659 & 3231 & 4816 & 9982 \\
\hline Xuño & 1537 & 2501 & 2378 & 3232 & 6415 \\
\hline Xullo & 1549 & 1771 & 1972 & 2375 & 6807 \\
\hline Agosto & 1726 & 1839 & 2032 & 2593 & 7981 \\
\hline Setembro & 2161 & 2305 & 6569 & 3821 & 7449 \\
\hline Outubro & 2385 & 2929 & 3489 & 3945 & 5282 \\
\hline Novembro & 2203 & 2682 & 3103 & 4193 & 3094 \\
\hline Decembro & 1796 & 2337 & 1893 & 1965 & 3676 \\
\hline Total & 22398 & 27932 & 33837 & 41096 & 85221 \\
\hline Media mes & 1866 & 2328 & 2820 & 3425 & 7102 \\
\hline
\end{tabular}

Nota: Elaboración por M.C. Guisán (2018) a partir de datos de RedAlyc.

Táboa 4. Descargas na base internacional Ideas.Repec.

\begin{tabular}{|l|c|c|}
\hline Ano & Descargas & Descargas por mes \\
\hline 2012 (5 meses) & 254 & 51 \\
\hline 2013 & 715 & 60 \\
\hline 2014 & 874 & 73 \\
\hline 2015 & 1324 & 110 \\
\hline 2016 & 1133 & 94 \\
\hline 2017 & 960 & 80 \\
\hline $2018(11$ meses) & 967 & 88 \\
\hline
\end{tabular}

Fonte: Elaboración por M.C. Guisán(2018) a partir de datos de Ideas.Repec. 
Dende o ano 2012 no que se incluíu a RGE (con artigos só dende o ano 2008) na base Ideas.Repec, houbo un número crecente de descargas, acadando xa un total acumulado de máis de 6000 descargas, situándose no posto 813 entre 3119 revistas científicas de Economía da devandta base, no percentil 27 por orde decrecente de consultas, e polo tanto ben localizada, malia que aínda dispón de poucos artigos na devandita base.

\section{Índice SCOPUS, valoración MIAR e difusión en bibliotecas}

A revista RGE ten un amplo recoñecemento e está incluída no prestixioso índice SCOPUS de Elsevier e nouttros índices de calidade científica.

Das máis de 150 revistas científicas de Economía incluídas no Catálogo seleccionado de Latindex, só un 10\% están presentes na base SCOPUS de Elsevier. 0 índice Scimago Journal Ranking (SJR) correspondente a devandita base, achega algunhas estatísticas de citas bibliográficas. A RGE aumentou moito as citas no período 2012-2018, pasando dun total acumulado de só 14 citas no período 2002 ata o ano 2012, a 53 citas acumuladas ata 2017 (cun incremento de 39).

No índice ICDS da base MIAR da Universitat de Barcelona, a revista acada un índice de 9.9 e sitúase entre as revistas de Economía, de España e do mundo, de maior puntuación no devandito índice.

$\mathrm{Na}$ Web da Revista consta a seguinte información con respecto á difusión da RGE en catálogos de bibliotecas: “15 bibliotecas universitarias británicas, 15 bibliotecas especializadas alemás, 20 bibliotecas universitarias españolas, máis de 200 bibliotecas universitarias dos Estados Unidos, e outras". Esa información refírese a anos anteriores á crise económica de 2008, pois con posterioridade a esa data a edición impresa limitouse e potenciouse a súa difusión electrónica.

\section{Outras revistas de investigación económica publicadas en Galicia, 2001- 2018}

A táboa 5 inclúe varias revistas de investigación de Economía Aplicada publicadas en Galicia no período 2001-2018, destacadas por ter acceso aberto a todos ou gran parte dos seus artigos e estar incluídas nunha ou varias das bases internacionais SCOPUS, RedAlyc, Econ-Lit e Ideas.Repec.

Táboa 5. Revistas de investigación de Economía Aplicada publicadas en Galicia

\begin{tabular}{|l|l|l|}
\hline Nome da revista & Director/a & Edita \\
\hline $\begin{array}{l}\text { Applied Econometrics and } \\
\text { International Development } \\
\text { (AEID) }\end{array}$ & $\begin{array}{l}\text { María-Carmen Guisán Seijas, } \\
\text { Catedrática USC }\end{array}$ & $\begin{array}{l}\text { Euro-American } \\
\text { Association, EAAEDS }\end{array}$ \\
\hline $\begin{array}{l}\text { Atlantic Review of Economics } \\
\text { (AROE) }\end{array}$ & $\begin{array}{l}\text { Fernando González Laxe, } \\
\text { Catedrático UDC }\end{array}$ & $\begin{array}{l}\text { Colegio Economistas } \\
\text { de A Coruña }\end{array}$ \\
\hline $\begin{array}{l}\text { Estudios Económicos de } \\
\text { Desarrollo Internacional } \\
\text { (EEDI) }\end{array}$ & $\begin{array}{l}\text { María-Carmen Guisán Seijas, } \\
\text { Catedrática USC }\end{array}$ & $\begin{array}{l}\text { Euro-American } \\
\text { Association, EAAEDS }\end{array}$ \\
\hline $\begin{array}{l}\text { European Journal of } \\
\text { Government and Economics } \\
\text { (EJGE) }\end{array}$ & $\begin{array}{l}\text { José Manuel Sánchez Santos } \\
\text { Profesor Titular UDC }\end{array}$ & UDC \\
\hline
\end{tabular}




\begin{tabular}{|l|l|l|}
\hline $\begin{array}{l}\text { Regional and Sectoral } \\
\text { Economic Studies (RSES) }\end{array}$ & $\begin{array}{l}\text { María-Carmen Guisán Seijas } \\
\text { Catedrática USC }\end{array}$ & $\begin{array}{l}\text { Euro-American } \\
\text { Association, EAAEDS }\end{array}$ \\
\hline $\begin{array}{l}\text { Revista Galega de Economía } \\
\text { (RGE) }\end{array}$ & $\begin{array}{l}\text { Directores do período 2001- } \\
\text { 2018 (ver Táboa 1) }\end{array}$ & USC \\
\hline $\begin{array}{l}\text { Urban Public Economic } \\
\text { Review (UPER) }\end{array}$ & Luis Caramés Viéitez & $\begin{array}{l}\text { Asociación de } \\
\text { Economía Pública } \\
\text { Urbana }\end{array}$ \\
\hline
\end{tabular}

Nota: EEDI publicouse no período 2001-2010 e está accesible na internet na Web da revista e en Ideas.Repec. UPER publicouse no período 2004-2015 e está accesible na internet a través de Dialnet. AEID, RSES publicadas dende 2001 e AROE, dende 2004, están accesibles dende a Web das revistas e en Ideas.Repec. A RGE está accesible, en versión galego dende 1992 e en castelán dende o ano 2000, a través da Web da revista: http://www.usc.es/econo/RGE/benvidag.htm

A Revista Galega de Economía é a máis antiga das revistas de investigación económica publicadas no período 2001-2018, xa que iniciou a súa publicación no ano 1992.

No Anexo incluímos unha referencia ás revistas de investigación económica de Galicia publicadas antes de 1992, e a outras do período 1992-2018.

\section{Investigación económica en Galicia e difusión social}

No Suplemento ao Volume 16-1 desta revista, inclúese o artigo de Barreiro (2007) sobre 15 anos de pensamento económico en Galicia, e unha lista de 102 nomes de autores de teses doutoriais do período 1992-2006, dos que 49 corresponden a mulleres (48\%) e 53 a homes (52\%). Os autores pertencen ás tres universidades de Galicia. Nese suplemento preséntase unha ampla información sobre autores e artigos da Revista Galega de Economía nese periodo. En Guisán (2018) inclúese información de moitas liñas de investigación económica desenvolvidas en Galicia.

Autores e Revistas de investigación económica de Galicia en bases internacionais

Na base Ideas.Repec Galicia ocupou no período lugares moi destacados, no 5\% do top de máis de 5000 institucións de todo o mundo polo número de traballos de investigación nesta base e polo número de consultas dos devanditos traballos. Varias autoras e autores destacan no $5 \%$ e o $10 \%$ de autores máis consultados na devandita base. No Anexo incluímos algunhas ligazóns interesantes neste senso.

Galicia ten unha boa presenza na base Scopus (con 3 revistas de Economía publicadas en Galicia entre as 15 revistas seleccionadas das máis de 150 revistas científicas españolas de Economía) e noutras importantes bases internacionais de revistas de investigación económica. Na base Ideas-Repec os investigadores de Galicia destacan, de forma que a USC figura no top $1 \%$ de institucións do mundo con traballos máis consultados nesa base internacional.

\section{Difusión social da investigación: Boletíns, Blogs, libros, prensa, TV e vídeos}

Difusión social: Conforme a Aguaded e Macías (2008) consideramos moi importante impulsar a divulgación da investigación económica de interese social realizada en Galicia. A RGE contribúe, na medida das súas posibilidades, a este obxectivo non só coa publicación gratuíta de artigos de investigación, senón tamén con iniciativas de divulgación. 
Boletíns e Blogs: Na Web do equipo de Econometría da USC hai unha sección do Boletín Económico da Asociación Hispalink-Galicia, na que incluímos comentarios e ligazóns sobre libros de divulgación científica da Economía, especialmente daqueles que están dispoñibles na Internet e outros documentos de divulgación da investigación económica de Galicia, tanto a nivel rexional, como nacional ou internacional.

Blog de Economía de Galicia: O Blog "Hispalink-Galicia”, coordinado pola Profesora Ma Carmen Guisán, inclúe entradas de divulgación de artigos publicados na Revista Galega de Economía, análises, noticias, datos económicos e ligazóns a outros Blogs.

En Econ-Academics, iniciativa de Blogs de Ideas.Repec, inclúense centos de Blogs académicos de todo o mundo, entre eles, os seguintes, publicados dende Galicia, con interesantes achegas para a divulgación científica da Economía. O Blog de Capital Social está coordinado pola Profesora Marta Portela e os outros tres pola Profesora Ma Carmen Guisán.

Capital Social: https://capitalsocialenespanol.wordpress.com/

Desarrollo de Galicia y España: https://economiaydesarrollo-eeg.blogspot.com.es

Economía Internacional: https://economiaydesarrollointernacional/blogspot.com.es

World Development: https://euroamericanassociation.blogspot.com.es

Libros de profesores universitarios: En Guisán (2018 a,b) incluímos unha relación de interesantes libros de divulgación da investigación económica publicados no período 1983-2018, dos que moi poucos están en edición electrónica aberta. Nas vindeiras actualizacóns do Anexo de Guisán (2018 a) agardamos ampliar a información sobre outros libros de interese.

Prensa, TV e Vídeos: Polo que respecta á prensa e á TV, a difusión da investigación universitaria de Economía é, en xeral, escasa en Europa. 0 Eurobarómetro indica que un $31 \%$ dos lectores de prensa está interesado na "investigación científica" e un $28 \%$ na "información económica", porcentaxes que indican un grao importante de interese social, sobre a investigación científica de Economía Aplicada ao desenvolvemento económico, moi por enriba da información que proporciona a meirande parte dos devanditos medios de comunicación. As universidades deberían propiciar unha maior presenza neses medios. Nos Blogs de economía que indicamos ao final da bibliografía, inclúense ligazóns relacionadas co obxectivo de difusión da investigación. Incluímos unha ligazón á Web Social-media-en investigación, que publica información de interese sobre a divulgación científica universitaria na internet. Estamos de acordo con Aguaded e Macías(2008) e outros autores que destacan a importancia da divulgación da investigación económica de interese social.

\section{Bibliografía}

Aguaded, I., Macías, Y. (2008). Televisión universitaria y servicio público. Revista Comunicar, Vol. XVI, no $31 .{ }^{1}$

Barreiro. X. (2007). A Revista Galega de Economía: quince anos de pensamento económico en Galicia. Revista Galega de Economía, Vol. 16-1, Suplemento. ${ }^{2}$

Guisán, M.C. (2015). "Informe 2015: 25 Años De Investigacion Económica En Galicia, 1967-1991" Revista Galega de Economía, Vol. 24-1, pp. 135-140.

Guisan, M.C., Exposito, P. (2007). "Fifty years of Econometrics Research in Spain: Doctoral Dissertations, International Publications and Other Contributions, 1956-2006". Working Paper of the Series Economic Development, no $98 .{ }^{5}$ 
Guisán, M.C. (2018 b). Investigación Económica En Galicia 1967-2017: Tesis Doctorales Y Estudios De Desarrollo Económico Regional, Sectorial e Internacional, Documento de la Serie Economic Development, no 121 . $^{1}$

1 http://www.usc.es/economet/econometria.htm

2 Boletín Económico da Asociación Hispalink-Galicia:

http://www.usc.es/economet/galicia.htm

${ }^{3}$ Blog da Asociación Hispalink Galicia

https://hispalinkgalicia.blogspot.com.es

Anexo. Revistas que iniciaron a súa publicación antes de 1992

$\mathrm{Na}$ segunda metade do século XX publicáronse varias revistas de Economía en Galicia, como as seguintes:

Revista de Economía de Galicia. Publicouse entre 1958-1968, pola Editorial Galaxia. O seu principal obxectivo era estudar a realidade socio-económica de Galicia. Máis información en: https://gl.wikipedia.org/wiki/Revista_de_Econom\%C3\%ADa_de_Galicia

Exemplares en Rebiun: http://catalogo.rebiun.org/

\begin{tabular}{|l|l|}
\hline $\begin{array}{l}\text { el } \\
\text { econarrol } \\
\text { de Galicia }\end{array}$ & $\begin{array}{l}\text { Rio Barja, Francisco. Bibliografía económica } \\
\text { de Galicia. Galaxia, Vigo, 1960 } \\
\text { http://consellodacultura.gal/404.php }\end{array}$ \\
\hline
\end{tabular}

\begin{tabular}{|c|c|}
\hline 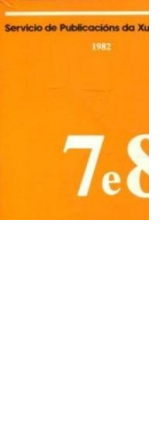 & $\begin{array}{l}\text { Revista Galega de Estudios Agrarios. 1979- } \\
\text { 1987. Xunta de Galicia. Non atopamos que } \\
\text { os artigos estén dispoñibles na internet. } \\
\text { http://iacobus.usc.es/search*gag/m?SEARCH=R } \\
\text { evista+Galega+de+Estudios+Agrarios\&sortdrop } \\
\text { down=-\&searchscope }=1 \\
\text { Ejemplares en Rebiun: } \\
\text { http://rebiun.baratz.es/rebiun/search?start=0\& } \\
\underline{\text { rows=12\&msp_reqtime=true\&fq=msstored_fld6 }} \\
\underline{\text { 6\&fv=CAT SERI\&q=Revista+Galega+de+Estudio }} \\
\underline{\text { s+Agrarios }}\end{array}$ \\
\hline
\end{tabular}

\begin{tabular}{|l|l|}
\hline $\begin{array}{l}\text { REVISTA } \\
\text { ECONOM } \\
\text { Y EMPRE }\end{array}$ & $\begin{array}{l}\text { Revista de Economía y Empresa. } \\
\text { Director en 1979: Camilo Prado Freire } \\
\text { Secretario en 1979: Justino Bastida }\end{array}$ \\
& $\begin{array}{l}\text { En Dialnet dende 1978 ata 2006 } \\
\text { https://dialnet.unirioja.es/servlet/revista?codig } \\
\end{array}$ \\
\hline
\end{tabular}

Revista Galega de Economía: http://www.usc.es/econo/RGE/benvidag.htm 